\title{
Évaluation du Risque Cardiovasculaire Absolu Chez les Patients Hémodialysés Diabétiques et Non diabétiques au Centre National d'Hémodialyse de Donka Conakry
}

\author{
Fousseny Diakité, \\ Alpha Koné, \\ Service de cardiologie hôpital national d'Ignace Deen \\ Mamadou Saliou Baldé, \\ Moussa Traoré, \\ Ibrahima Chérif, \\ Alpha Boubacar Bah, \\ Marcèlle Djamen Batchou, \\ Alpha Oumar Bah, \\ Mohamed Lamine Kaba,
} Service de néphrologie-hémodialyse, hôpital national de Donka

Service de néphrologie-hémodialyse, hôpital national de Donka

\section{Résumé}

Le risque cardiovasculaire chez les hémodialysés semble varier en fonction du statut diabétique ainsi que d'autres facteurs associés et constitue un problème de santé publique en Afrique en général et particulièrement en Guinée. Dans ce sens, l'objectif de cette étude était d'évaluer le risque de survenue d'une pathologie cardiovasculaire absolu chez les patients hémodialysés diabétiques, par rapport aux patients hémodialysés non diabétiques. La présente, étude transversale, descriptive et analytique a été réalisée entre le $1^{\text {ier }}$ avril et le 30 juin 2019 au Centre National d'Hémodialyse de Donka. L'étude a inclus les 140 patients hémodialysés durant la période. Le recrutement était exhaustif et concernait tous les patients hémodialysés répondant aux critères de sélection. Les données ont été recueillies prospectivement chez les patients hémodialysés puis compilées et traitées dans Epi info. 7.2.2.6. Un questionnaire semi-administré a été utilisé à ce fin. Pour évaluer le risque cardiovasculaire chez les patients le FRAMINGHAM RISK SCORE (FRS) a été aussi utilisé. L'enquête a concerné 140 individus dont 91 $(65,00 \%)$ étaient des hommes, contre $49(35,00 \%)$ de femmes, soit un sex- 
ratio de 1,86 soit 2 hommes pour une femme. L'âge moyen était de $41 \pm 4,1$ ans avec des extrêmes de $(30 ; 74)$ ans. On notait une prédominance chez les hommes des facteurs de risque, pour le tabagisme. L'étude a trouvé 39 diabétiques contre 101 non diabétiques. Le risque était élevé chez 23 diabétiques sur 39 ; et 25 sur 101 des patients non diabétiques. L'étude montre qu'un patient sur quatre aurait un risque absolu élevé chez les patients non diabétiques et un patient sur deux chez les patients diabétiques. Ce risque est majoré par d'autres facteurs associés.

Mots-clés: Risque absolu, Maladie cardiovasculaire, Hémodialysés, Conakry

\title{
Evaluation of Absolute Cardiovascular Risk in Diabetic and Non-Diabetic Hemodialysis Patients at the Donka Conakry National Hemodialysis Centre
}

\author{
Fousseny Diakité, \\ Service de néphrologie-hémodialyse, hôpital national de Donka \\ Alpha Koné, \\ Service de cardiologie hôpital national d'Ignace Deen \\ Mamadou Saliou Baldé, \\ Moussa Traoré, \\ Ibrahima Chérif, \\ Alpha Boubacar Bah, \\ Marcèlle Djamen Batchou, \\ Alpha Oumar Bah, \\ Mohamed Lamine Kaba, \\ Service de néphrologie-hémodialyse, hôpital national de Donka
}

\begin{abstract}
Introduction: Cardiovascular risk in hemodialysis appears to vary depending on diabetic status and other associated factors and is a public health problem in Africa in general and particularly in Guinea. The objective of this study was to assess the risk of absolute cardiovascular disease in diabetic hemodialysis patients, compared to non-diabetic hemodialysis patients. Methods: Between April 1 and June 30, 2019, a descriptive and analytical cross-sectional study was conducted at the National Hemodialysis Centre in
\end{abstract}


Donka. The study included 140 hemodialysis patients during the period. Recruitment was comprehensive and involved all hemodialysis patients meeting the selection criteria. The data were collected prospectively in hemodialysis patients and then compiled and processed in Epi info. 7.2.2.6. A semi-administered questionnaire had been used. To assess cardiovascular risk in patients, FRAMINGHAM RISK SCORE (FRS) was used. Results: The survey involved 140 individuals, 91 of whom (65.00\%) $49(35.00 \%)$ were men. sex ratio of 1.86 or 2 men per woman. The average age was 41-4.1 years with extremes of $(30 ; 74)$ years. There was a predominance among men of risk factors for smoking. The study found 39 diabetics versus 101 non-diabetics. The risk was high in 23 out of 39 diabetics; and 25 out of 101 non-diabetic patients. Conclusion: The study shows that one in four patients would have a high absolute risk in diabetic and non-diabetic patients and also confirms the association of other factors that increase this risk. The survey involved 140 individuals, 91 of whom $(65.00 \%) 49(35.00 \%)$ were men. sex ratio of 1.86 or 2 men per woman. The average age was 41-4.1 years with extremes of (30; 74) years. There was a predominance among men of risk factors for smoking and diabetes. In our series, most of our patients had a low risk level of 56 (40\%). Conclusion: This survey finds a low risk in this hemodialysis population. This should lead to strengthening strategies for preventing cardiovascular disease in this at-risk population.

Keywords: Absolute risk, Cardiovascular disease, Hemodialysis, Conakry

\section{Introduction}

Les maladies cardio-vasculaires, en particulier l'insuffisance coronarienne, représentent la première cause de morbidité et de mortalité dans les pays industrialisés. Le substratum de cette pathologie est une athérosclérose aboutissant à une plaque vulnérable et à un processus d'athérothrombose, cause des complications cliniques bien connues (infarctus du myocarde, thrombose cérébrale) dont beaucoup sont fatales ou résultent en invalidités définitives (Naghavi, Libby \& Falk, 2003). La prévention de ces maladies représente donc un problème de santé publique prioritaire depuis de nombreuses années (Pyörälä, De Backer \& Graham, 1996). Cette prévention ne sera efficace que s'il est possible de dépister facilement les individus à risque et d'instaurer des stratégies susceptibles de modifier radicalement l'histoire naturelle de la maladie. Plusieurs facteurs de risque ont été individualisés dans de nombreuses études épidémiologiques, dont la célèbre étude de Framingham entamée il y a plus de 40 ans (Dawber, Kannel \& Revotskie, 1962). Le risque cardio-vasculaire semble varier selon que le patient insuffisant rénal soit diabétique ou non, mais son caractère indépendant n'est pas attesté (Sarwar et al., 2007; Diallo et al., 2014). 
En Guinée, le nombre de patients hémodialysés est passé de 90 en 2015 à 140 en 2018 par augmentation du nombre de machines de dialyse. À cet égard, l'objectif de l'étude était déterminer la fréquence du risque cardio-vasculaire absolu ainsi que les facteurs y associés chez les hémodialysés du Centre National d'Hémodialyse de Donka.

\section{Patients et méthodes}

L'étude était prospective et comparative réalisée au Centre National d'Hémodialyse de Donka durant le 2ème trimestre de 2019.

Tous les patients hémodialysés suivis au Centre National d'Hémodialyse de l'Hôpital National Donka constituaient la population d'étude.

\section{Critère d'inclusion}

Patients hémodialysés adultes, homme et femme, ayant donné leur consentement éclairé.

Le recrutement était exhaustif et concernait tous les patients hémodialysés répondant aux critères de sélection.

Les données ont été recueillies prospectivement chez les patients hémodialysés puis compilées et traitées dans Epi info. 7.2.2.6. Un questionnaire semi-administré aété utilisé.

Pour évaluer le risque cardiovasculaire chez les patients le FRAMINGHAM RISK SCORE (FRS) a été utilisé. C'est un algorithme spécifique au sexe utilisé pour estimer le risque cardiovasculaire sur 10 ans d'un individu à partir des paramètres tels que l'âge, le sexe, le cholestérol total, le cholestérol HDL, le statut tabagique et la tension artérielle (systolique), en attribuant pour chaque paramètre un score qui lui est associé et en faisant la somme selon le sexe pour obtenir la prédiction du risque. Ce risque est ensuite classé en trois (03) catégories : faible (inférieur ou égal à 10\%), moyen (entre $10 \%$ et $19 \%$ ) ou élevé (supérieur ou égale à 20\%) (Mahmood et al., 2014; Wilson, 2013) voir à l'annexe l'interprétation.

Les variables étaient les suivantes : les caractéristiques sociodémographiques ; cliniques et paracliniques.

Un identifiant (Numéro) unique a été attribué aux patients afin de préserver l'anonymat de ces derniers. Seuls les médecins traitants et autres agents autorisés par le patient ont eu accès aux informations recueillies.

\section{Aspects éthiques}

Avant d'entreprendre toute activité sur le terrain, le protocole de recherche a été préalablement soumis à l'approbation de la chaire Néphrologie-Hématologie de la Faculté des Sciences et Techniques de la Santé de l'Université Gamal Abdel Nasser de Conakry. Sur le terrain, un consentement éclairé écrit a été également obtenu des patients inclus dans la 
présente étude. La participation à l'étude était totalement volontaire. Aucun patient ayant décliné d'y participer n'a été inclus. Chaque patient a signé ou apposé son empreinte digitale sur une fiche de consentement volontaire et éclairé qui lui a été lu par le candidat. Pour les patients incapables de lire la langue française, une traduction du contenu du formulaire de consentement éclairé a été faite en langue locale en présence d'un témoin ou membre de la famille.

\section{Résultats}

Le tableau I montre la fréquence élevée chez les hémodialysés âgés de 30 à 34 ans ; les plus de 70 ans ne représentaient que $2 \%$ environ.

Tableau I. Répartion des patients en fonction de l'âge

\begin{tabular}{|c|c|c|}
\hline Âge (années) & Fréquence & Pourcentage (\%) \\
\hline $30-34$ & 25 & 17,85 \\
\hline $35-39$ & 17 & $12,14 \%$ \\
\hline $40-44$ & 13 & $9,29 \%$ \\
\hline $45-49$ & 13 & $9,29 \%$ \\
\hline $50-54$ & 10 & $7,14 \%$ \\
\hline $55-59$ & 22 & $15,71 \%$ \\
\hline $60-64$ & 22 & $15,71 \%$ \\
\hline $65-69$ & 15 & $10,71 \%$ \\
\hline $70-74$ & 3 & $2,14 \%$ \\
\hline
\end{tabular}

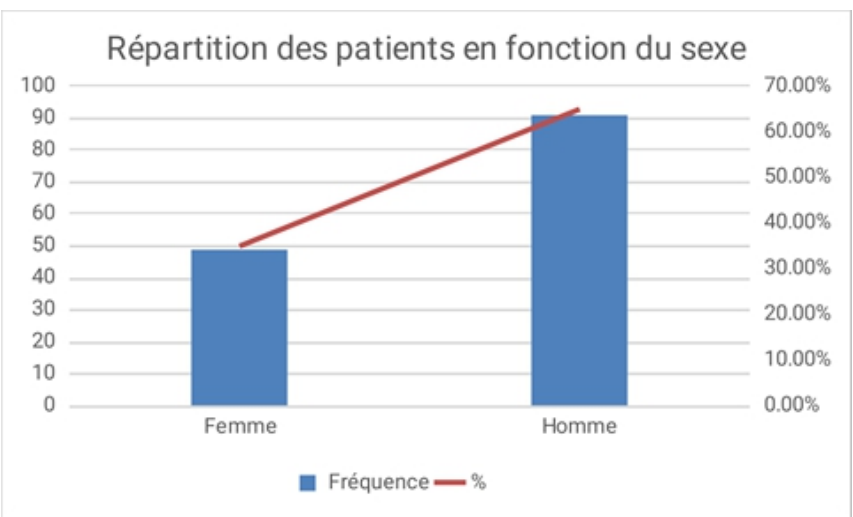

Selon cette figure les hommes étaient plus nombreux que les femmes. Le Tableau II montre une fréquence de risque élevée chez les hémodialysés $48 / 140$ et moyenne chez $36 / 140$. 
Tableau II. Répartition des patients en fonction du niveau de risque cardiovasculaire

\begin{tabular}{|c|l|l|}
\hline Niveau de risque (\%) & Fréquence & Pourcentage (\%) \\
\hline Faible & 56 & 40,00 \\
\hline Moyen & 36 & $25,71 \%$ \\
\hline Elevé & 48 & $34,29 \%$ \\
\hline TOTAL & 140 & $100,00 \%$ \\
\hline
\end{tabular}

Le Tableau III montre, parmi les 115 patients qui avaient au moins un risque, 98/115 avaient une pathologie cardiaque contre 17 pour les pathologies vasculaires.

Tableau III. Répartition des patients hémodialysés en fonction des pathologies cardiovasculaires

\begin{tabular}{|c|l|l|}
\hline Signes de cardiopathies & Fréquence & Pourcentage \\
\hline Accident vasculaire cérébrale & 2 & $1,74 \%$ \\
\hline Infarctus du myocarde & 3 & $\mathbf{2 , 6 1 \%}$ \\
\hline Insuffisance cardiaque congestive & 95 & $\mathbf{8 2 , 6 1} \%$ \\
\hline Vasculopathie périphérique & 15 & $13,04 \%$ \\
\hline TOTAL & 115 & $\mathbf{1 0 0 , 0 0} \%$ \\
\hline
\end{tabular}

Le tableau ci-dessous montre que l'âge, le cholestérol total, le cholestérol HDL et le niveau de risque sont des facteurs statistiquement significatifs dans cette étude.

Tableau IV. Résultats des tests statistiques effectués

\begin{tabular}{|l|l|l|l|l|}
\hline Variables & Chi-square & df & p-value & Effectif \\
\hline Age & 2.7134 & 8 & 0.0007 & 140 \\
\hline IMC & $\mathbf{3 . 0 8 1 6}$ & 5 & 0.6874 & 140 \\
\hline Cholestérol total & $\mathbf{9 . 4 1 8 1}$ & 4 & 0.0515 & 135 \\
\hline Cholestérol HDL & 12.5805 & 4 & 0.0131 & 135 \\
\hline Niveau de risque & 19.7248 & 2 & 0.0001 & 140 \\
\hline $\begin{array}{l}\text { Pathologies } \\
\text { cardiovasculaires }\end{array}$ & 6.2942 & 4 & 0.6981 & 140 \\
\hline
\end{tabular}

\section{Discussion}

Sur un effectif de 140 patients hémodialysés, la tranche d'âge la plus représentée était celle de 30 à 34 ans avec 25 cas soit 17,86\%, et la moyenne d'âge était de 41 ans $\pm 10 \%$. Les résultats sont différents de ceux de

Pessinaba, Mbaye, et Yabéta (2013) qui avaient retrouvé un âge moyen de 53,6 \pm 15,9 ans avec des extrêmes : 16 ans et 96 ans.

L'étude avait monté une prédominance masculine $65 \%$ contre $35 \%$ des femmes.

Edgar et al. (2011) ont eu un sex-ratio inverse au celuid e cette étude avec plus de femmes que d'hommes.

Les patients diabétiques représentaient 39 (27,86\%), contre 101 (72,14\%) non diabétiques. Au Sénégal en 2010, 17 cas $(10 \%)$ avaient été obtenus (Mbaye, Niang \& Sarr, 2011). 
L'étude montre un niveau de risque élevé soit 48/140 (34.28\%) ; 40\% des patients avait un risque faible. Cela concorde avec les résultats du modèle de Framingham (24,9\%), de la société Européenne d'Hypertension (28\%) et celui du programme SCORE $(6 \%)$.

L'étude a retrouvé une différence statistiquement significative entre les différents niveaux de risque selon le statut diabétique. Le statut diabétique influence négativement le risque cardiovasculaire $\mathrm{Chi}^{2}=19,7248$, et $\mathrm{P}$-value $=0,0001$. Bouattar et al. (2009) en 2010 au Maroc, avaient constaté un résultat similaire ( $\mathrm{P}$-value $=0,0002)$ et qui en plus avait quantifié ce risque pour conclure que les patients hémodialysés diabétiques étaient trois fois plus exposés aux risques de survenue de maladies cardiovasculaires que les patients hémodialysés non diabétiques.

Sur un échantillon de 140 patients, 115 présentaient au moins un signe de pathologie cardiovasculaire soit environ $82,14 \%$.

Une différence statistiquement significative de distribution des pathologies cardiovasculaires a été retrouvée chez les patients indépendamment du sexe, qu'ils aient été diabétique ou non $\left(\mathrm{Chi}^{2}=6,2942\right)$ ou (P-value $=0,0981)$. Le contraire a été observé dans l'étude de Bouattar et al. (2009) qui en 2010 au Maroc, avaient retrouvé plus de pathologies cardiovasculaires chez les patients hémodialysés diabétiques par rapport aux patients hémodialysés non diabétiques.

\section{Conclusion}

Le risque absolu était élevé chez les patients hémodialysés. Les principaux facteurs associés étaient : l'âge,le cholestérol et le niveau de risque cardiovasculaire Il conviendrait dans un avenir proche, de mettre en place un ensemble de mesures préventives et de prise en charge afin de réduire l'impact de ce risque dans les populations à risque qui constitue une source de dépense non négligeable.

\section{Limites de l'étude}

- Le niveau socio-économique des patients pour répéter parfois les examens.

- La mesure de certains facteurs de risque cardiovasculaire (tabac) basée sur des déclarations.

- La réalisation des examens biologiques dans des laboratoires différents.

\section{Conflit d'intérêt}

Les auteurs déclarent qu'il n'y a aucun conflit d'intérêt lié à cette étude. 


\section{References:}

1. Bouattar, T., Benasila, S., \& Mattous, M. (2009). L'atteinte rénale chez le diabétique âgé. Elsevier Masson SAS. doi:10.1016/j.npg.2009.12.001.

2. Dawber, TR., Kannel, W., \& Revotskie, N. (1962). The epidemiology of coronary heart disease - the Framingham Enquiry. Proc Roy Soc Med, 55, 265-271.

3. Diallo, M., Diallo, A., Balde, N., Camara, A., Bah, A., Bah, A. (2014). P339 Profil épidémiologique, clinique et thérapeutique de 100 diabétiques suivis en ambulatoire au CHU de Conakry, en Guinée. Diabetes \& Metabolism. mars; 40:A108. Disponible sur: https://linkinghub.elsevier.com/retrieve/pii/S1262363614726308

4. Mahmood, SS., Levy, D., Vasan, RS., \& Wang, TJ. (2014). The Framingham Heart Study and the epidemiology of cardiovascular disease: a historical perspective [archive], Lancet; 383:999-1008.

5. Mbaye, M.N., Niang, K., \& Sarr, A. (2011). Aspects épidémiologiques du diabète au Sénégal : résultats d'une enquête sur les facteurs de risque cardiovasculaire dans la ville de Saint-Louis. Médecine des maladies Métaboliques - Décembre - Vol. 5 - $\mathrm{N}^{\circ} 6$.

6. Naghavi, M., Libby, P., \& Falk, E. (2003). From vulnerable plaque to vulnerable patient. A call for new definitions and risk assessment strategies. Circulation 108, 1664-1672 (part I) and 1772-1778 (part II).

7. Ngoungou, E. B., Aboyans, V., \& Kouna, P. (2011). Prévalence des affections cardiovasculaires en population générale au Gabon. Clinical Research. doi: 10.1016/j.acvd.2011.12.005.

8. Peter, W., Wilson, F., Ralph, B., D'Agostino, \& Daniel, L. (1998). "Prediction of coronary heart disease using risk factor categories". Circulation. $\quad 97 \quad$ (18)

$1837-1847$. https://doi.org/10.1161/01.CIR.97.18.1837

9. Pyörälä, K., De Backer, G., \& Graham, I. (1996). Prévention de la maladie coronarienne en pratique clinique : résumé pour les cliniciens. Rev Med Liège, 51, 654-660.

10. Pessinaba, S., Mbaye, A., \& Yabéta, G.A.D. (2013). Enquête de prévalence des facteurs de risque cardiovasculaire en population générale à Saint-Louis (Sénégal). Annales de Cardiologie et d'Angéiologie 62, 253-258.

11. Ralph, B., D’Agostino, S.R., \& Ramachandran, S. (2008). "General cardiovascular risk profile for use in primary care: the Framingham Heart Study" .Circulation; 117;743-753; originally published online Jan 22, DOI: 10.1161/CIRCULATIONAHA.107.699579.

12. Sarwar, N., Danesh, J., Eiriksdottir, G., Sigurdsson, G., Wareham, N., \& Bingham, S. (2007). Triglycerides and the Risk of Coronary Heart 
Disease. Circulation. 30 janv;115(4):450-8. Disponible sur: https://www.ahajournals.org/doi/abs/10.1161/circulationaha.106.637 793

\section{Annexe}

\section{Pour un homme}

○ Âge (en année) : 20-34 ans = moins 9 points, $35-39$ ans $=$ moins 4 points, $40-44$ ans $=0$ point, $45-49 \mathrm{ans}=3$ points, $50-54$ ans $=6$ points, $55-59$ ans $=8$ points, $60-64$ ans $=10$ points, $65-69$ ans $=11$ points, $70-$ 74 ans $=12$ points, $75-79$ ans $=13$ points.

○ Cholestérol total (mg/dl) : De 20 à 39 ans (moins de 160: 0 point, 160-199: 4 points, 200-239: 7 points, 240-279: 9 points, 280 ou plus: 11 points). De 40 à 49 ans: (moins de $160: 0$ point, 160-199: 3 points, 200-239: 5 points, 240-279: 6 points, 280 ou plus: 8 points). De 50 à 59 ans: (moins de $160: 0$ point, 160-199: 2 points, 200-239: 3 points, 240-279: 4 points, 280 ou plus: 5 points). De 60 à 69 ans: (moins de 160 : 0 point. $160-199: 1$ point, 200-239: 1 point, 240-279: 2 points, 280 ou plus: 3 points). De 70 à 79 ans: moins de $160: 0$ point, 160199: 0 point, 200-239: 0 point, 240-279: 1 point, 280 ou plus: 1 point).

○ Fumeur : De 20-39 ans: 8 points, 40 à 49 ans: 5 points, 50 à 59 ans: 3 points, $60-69$ ans: 1 point, $70-79$ ans: 1 point.

○ Non-fumeur : 0 point.

○ Cholestérol HDL (mg/dl) : 60 ou plus: Moins 1 point, 50-59: 0 point, 40-49: 1 point, Moins de 40 ans: 2 points

○ Tension artérielle systolique $(\mathrm{mm} / \mathrm{Hg})$ :

- Non traité: Moins de 120: 0 point, 120-129: 0 point, 130-139: 1 point, 140-159: 1 point, 160 ou plus: 2 points,

- Traité: Moins de 120: 0 point, 120-129: 1 point, 130-139: 2 points, 140-159: 2 points, 160 ou plus: 3 points,

Interprétation du Risque sur 10 ans (\%) (Ralph, D'Agostino \& Ramachandran, 2008): Total de points: 0 point: <1\%, 1-4 points: $1 \%, 5-6$ points: $2 \%, 7$ points: $3 \%, 8$ points: $4 \%, 9$ points: $5 \%, 10$ points: $6 \%, 11$ points: 8\%, 12 points: $10 \%, 13$ points: $12 \%, 14$ points: $16 \%, 15$ points: $20 \%, 16$ points: $25 \%, 17$ points ou plus: plus de $30 \%$.

\section{Pour une femme}

○ Âge (en année) : 20 à 34 ans: Moins 7 points, 35-39 ans: moins 3 points, $40-44$ ans: 0 point, $45-49$ ans: 3 points, $50-54$ ans: 6 points, 55-59 ans: 8 points, $60-64$ ans: 10 points, $65-69$ ans: 12 points, $70-74$ ans: 14 points, $75-79$ ans: 16 points,

○ Cholestérol total (mg/dl) : De 20 à 39 ans: moins de $160: 0$ point, 160-199: 4 points, 200-239: 8 points, 240-279: 11 points, 280 ou plus: 13 points, De 40 à 49 ans: moins de 160 : 0 point, 160-199: 3 points, 
200-239: 6 points, 240-279: 8 points, 280 ou plus: 10 points, De 50 à 59 ans: moins de $160: 0$ point, 160-199: 2 points, 200-239: 4 points, 240-279: 5 points, 280 ou plus: 7 points, De 60 à 69 ans: moins de 160 : 0 point, 160-199: 1 point, 200-239: 2 points, 240-279: 3 points, 280 ou plus: 4 points, De 70 à 79 ans: moins de $160: 0$ point, 160-199: 1 point, 200-239: 1 point, 240-279: 2 points, 280 ou plus: 2 points,

- Fumeuse : De 20-39 ans: 9 points, 40 à 49 ans: 7 points, 50 à 59 ans: 4 points, $60-69$ ans: 2 points, $70-79$ ans: 1 point,

$\circ$ Non fumeuse : 0 point,

○ Cholestérol HDL (mg/dl) : 60 ou plus: Moins 1 point, 50-59: 0 points, 40-49: 1 point, Moins de 40 ans: 2 points,

○ Tension artérielle systolique $(\mathrm{mm} / \mathrm{Hg})$ :

- Non traitée: Moins de 120: 0 points, 120-129: 1 point, 130139: 2 point, 140-159: 3 points, 160 ou plus: 4 points,

- Traitée: Moins de 120: 0 point, 120-129: 3 points, 130-139: 4 points, 140-159: 5 points, 160 ou plus: 6 points,

- Interprétation du Risque sur 10 ans (\%) (Ralph, D'Agostino \& Ramachandran, 2008): Total de points: Moins de 9 points: <1\%, 9 12 points: $1 \%, 13-14$ points: $2 \%, 15$ points: $3 \%, 16$ points: $4 \%, 17$ points: $5 \%, 18$ points: $6 \%, 19$ points: $8 \%, 20$ points: $11 \%, 21=14 \%$, $22=17 \%, 23=22 \%, 24=27 \%,>25=$ plus de $30 \%$. 